\title{
Influence of Spanwise Boundary Conditions on Slat Noise Simulations
}

\author{
David P. Lockard, ${ }^{*}$ Meelan M. Choudhari ${ }^{\dagger}$ and Pieter G. Buning ${ }^{\dagger}$ \\ NASA Langley Research Center, Hampton, VA 23681 USA
}

\begin{abstract}
The slat noise from the 30P/30N high-lift system is being investigated through computational fluid dynamics simulations with the OVERFLOW code in conjunction with a Ffowes Williams-Hawkings acousties solver. In the present study, two different spanwise grids are being used to investigate the effect of the spanwise extent and periodicity on the near-field unsteady structures and radiated noise. The baseline grid with periodic boundary conditions has a short span equal to 1/9th of the stowed chord, whereas the other, longer span grid adds stretched grids on both sides of the core, baseline grid to allow inviscid surface boundary conditions at both ends. The results indicate that the near-field mean statisties obtained using the two grids are similar to each other, as are the directivity and spectral shapes of the radiated noise. However, periodicity forces all acoustic waves with less than one wavelength across the span to be two-dimensional, without any variation in the span. The spanwise coherence of the acoustic waves is what is needed to make estimates of the noise that would be radiated from realistic span lengths. Simulations with periodic conditions need spans of at least six slat chords to allow spanwise variation in the low-frequencies associated with the peak of broadband slat noise. Even then, the full influence of the periodicity is unclear, so employing grids with a fine, central region and highly stretched meshes that go to slip walls may be a more efficient means of capturing the spanwise decorrelation of low-frequency acoustic phenomena.
\end{abstract}

\section{Nomenclature}

$\begin{array}{ll}a & \text { speed of sound } \\ C_{p} & \text { coefficient of pressure } \\ c & \text { stowed chord } \\ c_{s} & \text { slat chord } \\ d t & \text { time step } \\ f & \text { frequency } \\ L_{c} & \text { coherence length scale } \\ L_{s} & \text { span used in } \mathrm{FW}-\mathrm{H} \\ M & \text { Mach number, }\left|\mathrm{V}_{o}\right| / a_{o} \\ p & \text { pressure } \\ \mathrm{PSD} & \text { power spectral density }(\mathrm{dB} / \mathrm{Hz}) \\ \mathrm{Re}_{c} & \text { Reynolds number, }\left|\mathrm{V}_{o}\right| c / \nu_{o} \\ R_{p p} & \text { spanwise correlation of pressure } \\ \mathrm{rms} & \text { root mean square } \\ S t & \text { Strouhal number, } f^{*} c_{s} / U_{o} \\ \mathrm{TKE} & \text { turbulence kinetic energy } \\ u, v, w & \text { instantaneous velocity components } \\ U, V, W & \text { time-averaged velocity components } \\ |\mathrm{V}| & \text { magnitude of velocity vector }\end{array}$

$\begin{array}{ll}\left|\mathbf{V}_{2 D}\right| & \text { planar velocity mangitude } \\ x, y, z & \text { Cartesian coordinates }\end{array}$

Greek:

$\gamma^{2}(f) \quad$ spanwise coherence

$\rho \quad$ fluid density

$\Delta z \quad$ spanwise separation distance

$\Omega \quad$ vorticity

\section{Superseript:}

perturbation quantity (e.g., $\rho^{\prime}=\rho-\rho_{\infty}$ )

* dimensional quantity

Subscript:

$\infty \quad$ dimensionless free-stream quantity

- dimensional reference quantity

Operators:

<> time average (e.g., $U=<u>$ )

\section{Introduction}

The non-propulsive (or airframe) sources of aircraft noise include high-lift devices (e.g., the leading-edge slat and trailing-edge flaps) and the aircraft undercarriage. The ranking of these sources is configuration dependent; however, both model-scale tests ${ }^{1-7}$ and flyover noise measurements ${ }^{8}$ have identified the leading-edge slat as a prominent source of airframe noise during aircraft approach. The reduction of aircraft noise, including that of the airframe, is an important

\footnotetext{
*Aerospace Technologist, Computational AeroSciences Branch, Mail Stop 128, Senior Member, AIAA

${ }^{\dagger}$ Aerospace Technologist, Computational AeroSciences Branch, Mail Stop 128, Associate Fellow, AIAA
} 
goal of the NASA Advanced Air Transport Technology (AATT) Project, which is supporting a combined experimental and computational effort to better understand the sources associated with slat noise.

The near-field fluctuations within the slat cove have been investigated experimentally ${ }^{9}$ by using the 30P/30N model. The 30P/30N model tested in the Basic Aerodynamic Research Tunnel (BART) at NASA Langley Research Center represents a generic, three-element, zero-sweep high-lift configuration with slat and flap deflections of 30 degrees each. The slat chord and flap chord of the model are equal to $15 \%$ and $30 \%$, respectively, of the 18 " $(0.457 \mathrm{~m})$ stowed chord, c. For the approach configuration, the slat gap is $2.95 \%$; the flap gap is $1.27 \%$; and the slat and flap overhang settings are equal to $-2.95 \%$ and $0.25 \%$ of the stowed chord, respectively. Reference 10 provides a definition of these rigging parameters. At the test Mach number of 0.17, the Reynolds number based on the stowed chord of the BART model, $\operatorname{Re}_{c}$, is 1.71 million. While this Reynolds number is substantially lower in comparison with full-scale applications, the BART experiment is still suitable for validating numerical predictions of slat cove flow features that may generate noise as described in Ref. 11. New experiments at Florida State University ${ }^{12}$ and the Japanese Aerospace Exploration Agency ${ }^{13}$ have recently been reported that include unsteady surface pressure measurements along streamwise and spanwise rows of sensors.

All of these experiments as well as previous computational studies have found that the hydrodynamic features in the slat cove decorrelate rapidly, approximately within the length of the slat chord. Using CFD, the effect of the spanwise extent of the computational domain was investigated by Lockard and Choudhari ${ }^{14}$ by increasing the span from $c / 18$ (37.03\% of the slat chord $c_{s}$ or 1", as used in Ref. 11) to $c / 3\left(222 \%\right.$ of $c_{s}$ or 6") while maintaining the same spanwise resolution. The simulations with the longer span indicated that the spanwise surface pressure correlations do not become smaller than 0.05 until around $c / 9\left(74.06 \%\right.$ of $c_{s}$ or 2"). However, the spanwise correlation length of the acoustic signals, in regions where acoustic waves are easy to detect such as on the upper surface of the slat, was found to be on the order of a slat chord. Since multiple spanwise correlations must be included to directly compute the radiating noise, a much longer span than even $c / 3$ would be needed. Nonetheless, the near-field unsteadiness that gives rise to the noise should be adequately modeled with a $c / 9$ span.

For computational efficiency, numerical simulations have typically employed a relatively short span length with periodic boundary conditions. An OVERFLOW calculation ${ }^{15}$ of such a configuration suffered from an instability at the frequency corresponding to one acoustic wavelength across the spanwise domain. Periodicity is commonly employed in Large Eddy Simulations, but Bisek ${ }^{16}$ and Dawson ${ }^{17}$ recently found that periodicity had some adverse influence on compression ramp flow simulations, although nothing resembling the instability observed in the OVERFLOW calculation. Periodic conditions are assumed to have a minimal impact on the development of the basic flow features as long as sufficient spanwise decorrelation occurs within one half of the spanwise domain width. Although the hydrodynamic features around a slat meet this criteria, acoustic waves, which have much longer wavelengths for the same frequency, are often fully correlated across the entire span of a numerical simulation. Therefore, acoustic waves will resemble waveguide modes with cut on and cut off behavior that is consistent with the spanwise boundary conditions used in the simulations. The acoustic predictions from these simulations are usually obtained from an acoustic analogy where the surface pressure is used to drive a source term for a wave equation. If the hydrodynamic pressure footprint acts as the source, then errors in the acoustics induced by the periodic conditions could be ignored under the assumption that their amplitude is relatively small in comparison with the hydrodynamic components. However, the acoustic analogy can also be viewed as a filter that removes the hydrodynamic portion of the signal and extends the underlying acoustics. With this interpretation, any modification to the near-field acoustics would influence the far-field predictions. Hence, a numerical evaluation of the noise from configurations with and without periodic boundary conditions would help to assess whether the use of periodicity is appropriate in aeroacoustic simulations. To that end, we simulate a relatively large-span model (with an aspect ratio that is comparable to that in a typical wind tunnel test) with inviscid wall conditions at the spanwise boundaries. Note that this approach does not capture end-wall effects associated with viscous flow dynamics in that region.

\section{Computational Simulations}

The numerical study is being performed with the OVERFLOW ${ }^{18}$ code developed at NASA Ames Research Center. A previous simulation ${ }^{15}$ of the $30 \mathrm{P} / 30 \mathrm{~N}$ high-lift configuration was conducted with OVERFLOW using fifth-order spatial operators for the convective terms and shown to be in good agreement with a second-order, CFL $3 \mathrm{D}^{19}$ simulation on a refined grid. The spanwise extent was $c / 9$. The OVERFLOW calculation suffered from an instability at the frequency corresponding to one acoustic wavelength across the domain. Enough data was collected before the instability overwhelmed the solution, but this difficulty underscores the need for the current investigation.

The OVERFLOW ${ }^{18}$ calculation uses a fifth-order upwind spatial discretization with the Roe flux scheme for the 
inviscid terms on the right hand side. The viscous terms are still discretized to second order, as are the grid metrics and interpolation across overset meshes, so the code remains formally second order. However, on the smoothly varying meshes employed in this work, the fifth-order spatial operators produce solutions with much better dispersion and dissipation characteristics than low-order operators. OVERFLOW employs a dual-time-stepping algorithm where subiterations are used to converge the solution within each time step. The new calculation with an extended span uses 30 subiterations per time step to reduce the L2-norm of the flow equation residual by a minimum of two orders of magnitude. The time step in the current work is $d t^{*} a_{o} / c=0.0008161$ or $d t^{*}\left|\mathbf{V}_{\circ}\right| / c=0.0001379$, which results in 1,006 time steps per period at a frequency of $1 \mathrm{kHz}$ (Strouhal number, $S t=f^{*} c_{s} / U_{o}=1.18$ ). The corresponding convective scale for a particle traveling with the flow to traverse the stowed chord of the airfoil is 7,146 time steps. The left-hand-side operator of the inner iteration within the dual-time stepping algorithm involves a successive-over-relaxation scheme ${ }^{20}$ which is computationally intensive but has better convergence properties than many of the other operators implemented in the code. The previous, short span calculation ${ }^{15}$ used twice the time step with the ARC3D diagonalized Beam-Warming scalar pentadiagonal scheme ${ }^{21}$ and 40 subiterations. The changes were made in an effort to improve both computational efficiency and the convergence within each time step.

A zonal turbulence model was employed with pure RANS being used around the flap and a Delayed Multi-Scale Shear Layer Transport (SST) model ${ }^{20}$ over the rest of the domain. The Delayed Multi-Scale method is a Delayed Detached Eddy Simulation ${ }^{22}$ (DDES) variant for the SST turbulence model that was introduced by Strelets ${ }^{23}$ to overcome certain limitations of the original methodology for computational grids where the grid spacing tangential to viscous surfaces becomes smaller than the boundary layer thickness. DDES methodology employs RANS in near-wall regions where grid and time-step requirements make it impractical to resolve the small-scale boundary layer unsteadiness that is not expected to have a large impact on the outer flow that is dominated by larger scales. The model transitions from RANS to LES based on a switch that depends on the distance from the wall, the grid, and flow parameters.

\section{II.A. Configurations and Grids}

The $30 \mathrm{P} / 30 \mathrm{~N}^{10}$ high-lift system was simulated in free air without wind-tunnel walls above and below the airfoil. The simulations use an extruded 2-D geometry (in the nondimensional $x-y$ plane, with $c$ as the reference length). The freestream Mach number is 0.17 , and the Reynolds number is 1.71 million based on the stowed chord.

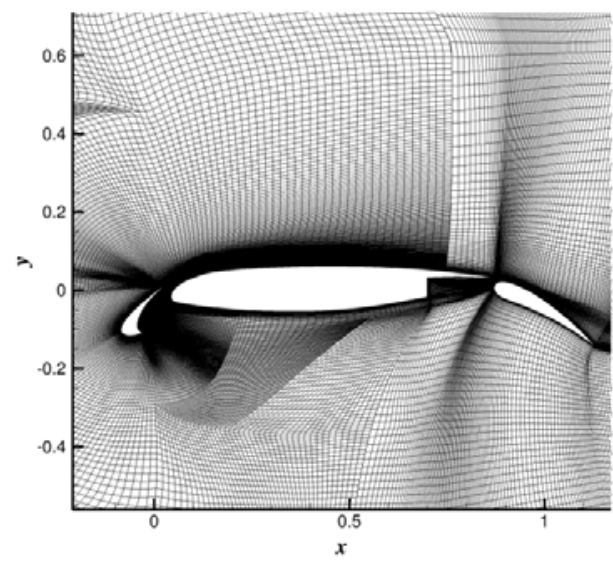

(a) Full airfoil view

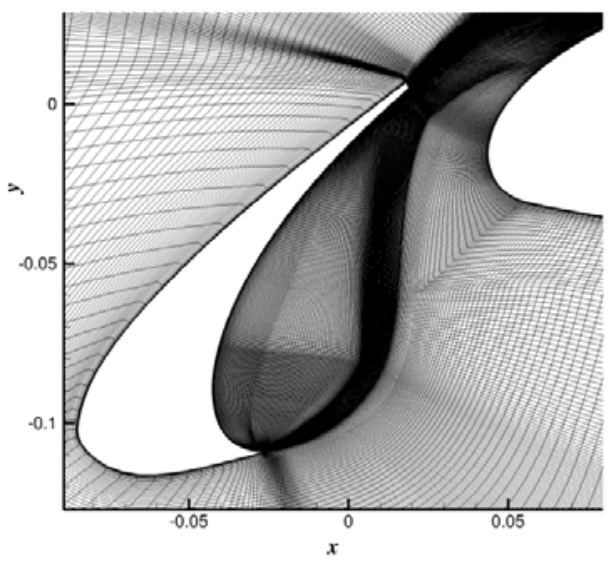

(b) Close-up of slat

Figure 1. Planar view of grid around the slat (every other point).

The investigated configuration corresponds to a free-flight configuration at $5.5^{\circ}$ angle of attack, which is approximately consistent with the mean slat loading for a $4^{\circ}$ angle of attack in BART. ${ }^{9}$ The block-structured, $x-y$ planar grid shown in Fig. 1(a) has 667,000 points in 32 blocks. Care was taken so that the first point off the solid surfaces was at $y^{+}<1$. Fig. 1(b) shows a close-up view of the grid in the vicinity of the slat, showing the clustering around the shear layer in the cove. The grid in a previous simulation ${ }^{15}$ had 129 points in the spanwise direction for a total of 86 million points.

An ideal simulation approach would involve using a uniform spanwise resolution over a spanwise domain width of approximately $2 c$ (a value typical of experimental tests), but the computational cost would be prohibitive. As an alternative, a relatively short spanwise uniform grid region is extended with a highly stretched mesh on both sides 
to obtain the same spanwise extent of $2 c$. Although the stretched mesh will dramatically affect the hydrodynamic features in the side regions, the acoustic waves with their longer wavelengths should be less affected as long as the largest spanwise spacing is less than $1 / M_{\infty}$ times the spacing in the core region. Furthermore, eliminating the periodic assumption allows flow features to decorrelate across the entire span, even if that is only meaningful in the uniform grid region. When periodic boundary conditions are employed, the data over half of the span must be ignored when computing parameters such as the spanwise coherence.

The spanwise domain in the new calculation is $2.9 c$ (52.2"). The center 129 spanwise points over $c / 9$ (2") of the grid are nearly identical to those employed in the previous simulation ${ }^{15}$ as shown in Fig. 2 where the spanwise distributions of the two grids are superimposed. The grid outside of this core region expands using a hyperbolic sine stretching function yielding a total of 225 points. The spanwise grid distribution was run through a high-order smoother to reduce discontinuities in higher derivatives of the grid metrics across the stretched/unstretched interfaces.

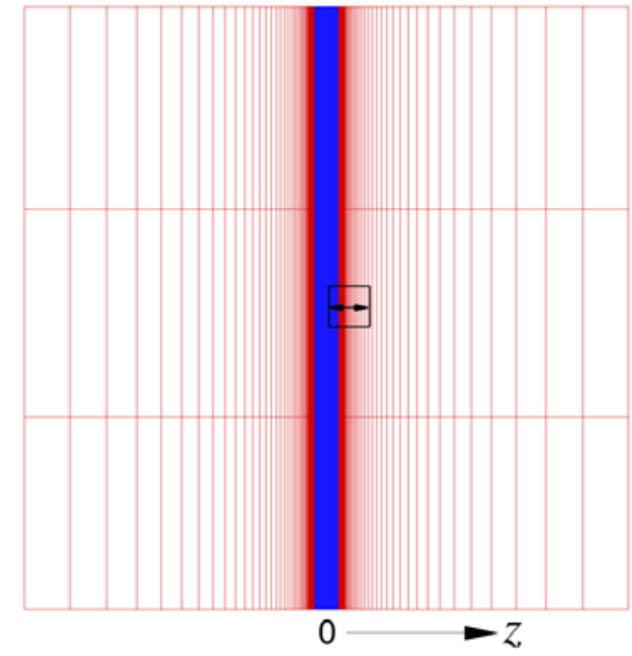

(a) Full-span view

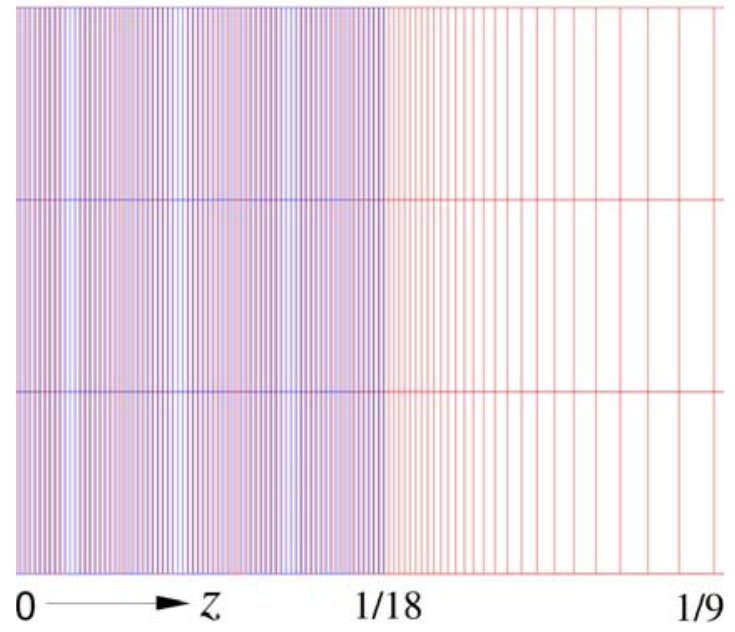

(b) Close-up of half span

Figure 2. Close-up in the $(x-z)$ plane showing the spanwise $(z)$ distribution of the grid. The original grid with a span of $c=9$ is in blue, and the new long-span grid with a span of $2: 9 \mathrm{c}$ is in red. The black box in (a) shows the spanwise extent in (b).

Characteristic boundary conditions were used along the far-field boundaries in the $x-y$ plane. The circular outer boundary was located 25 airfoil chords from a point in the slat cove. Inviscid boundary conditions were used at the spanwise boundaries of the computational domain in the new calculation instead of periodicity. No attempt was made to resolve the end effects associated with the presence of tunnel side walls in the experiments. No-slip conditions were imposed at the airfoil surfaces, along with an adiabatic wall thermal boundary condition.

\section{Near-Field Results}

The time-averaged coefficient of pressure, $C_{p}=<2\left(p^{*}-p_{o}\right) /\left(\rho_{o} U_{o}^{2}\right)>$, on the solid surfaces is presented in Fig. 3. Results are presented from the previous calculation ${ }^{15}$ with a span of $c / 9$, and the new calculation with a span of $2.9 c$. The data was generated by averaging over time and then averaging over the span by assuming that the flow is homogeneous in the spanwise direction. For the new calculation, only the center $c / 9$ of the grid was used in the spanwise average. The temporal duration of the signals is approximately $0.09 \mathrm{~s}$ for both simulations, which is sufficient for the flow to traverse the chord of the airfoil 11 times. Overall, the agreement is fairly good, although the new grid produces slightly lower suction values on the upper surface of the flap because the flap separation occurs a little earlier. The location of the flap separation was previously found to be sensitive to the turbulence model, ${ }^{15}$ so the variation observed with the change in span is not surprising.

A comparison of the calculated $C p_{r m s}^{\prime}$ is shown in Figs. 4 and 5. The $C p_{r m s}^{\prime}$ on the flap is somewhat different, which is to be expected based on the differences observed in the mean loading. Despite forcing RANS around the flap, both calculations still support some unsteadiness in that region because of the separation around the flap.

A close-up of $C p_{r m s}^{\prime}$ over the slat is shown in Fig. 5. The location where the fluctuations initially reach a maximum near the reattachment point in the slat cove between $x^{*} / c=0$ and 0.005 is slightly further upstream in the longer span 
calculation, probably because of the different mean flows. Otherwise, the curves are remarkably similar in terms of shape and levels.

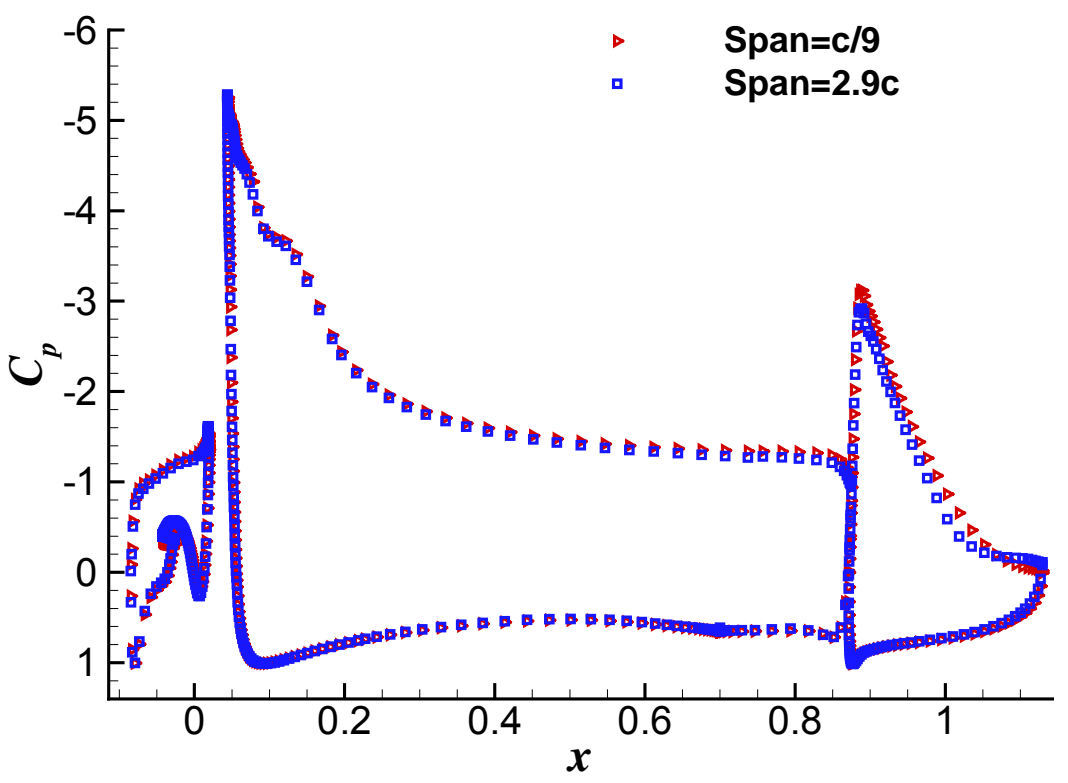

Figure 3. Time-averaged coefficient of pressure on the airfoil.

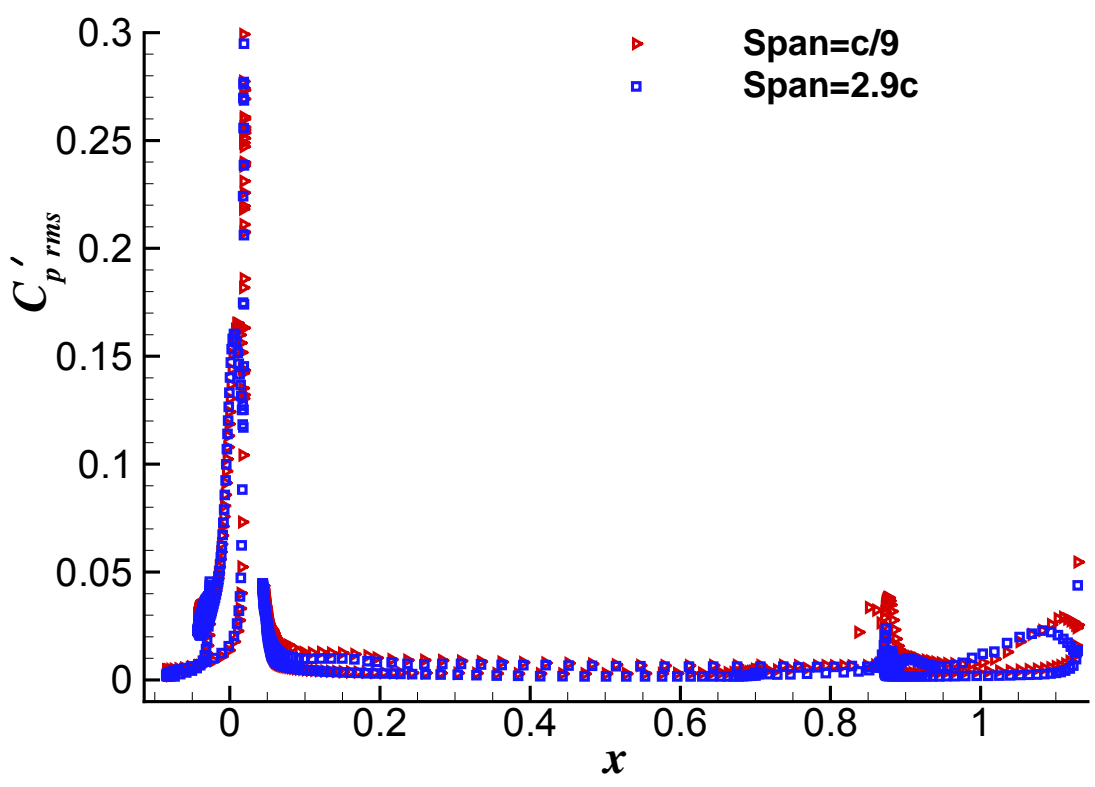

Figure 4. RMS of the fluctuating coefficient of pressure on the airfoil.

Contours of the magnitude of the mean planar velocity $\left|\mathbf{V}_{2 D}^{*}\right|$, nondimensionalized by $U_{o}$, are presented in Fig. 6. The points and lines identify locations for more detailed comparisons. The velocity distributions are visually similar for the two computations, but the center of the recirculation zone is shifted upward in the longer span case. The trajectory of the shear layer is also nominally the same (except for a slightly upstream reattachment for the span $=2.9 c$ case as indicated by the purple streamline superimposed in the figures). 


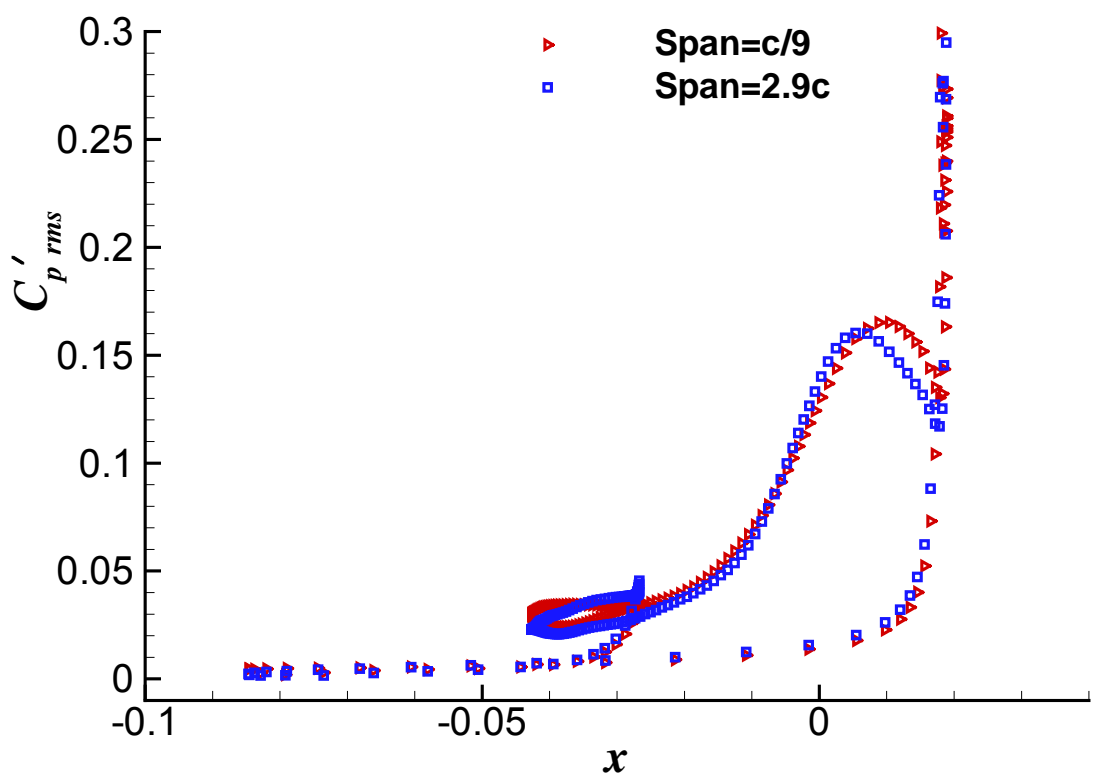

Figure 5. RMS of the fluctuating coefficient of pressure on the slat.

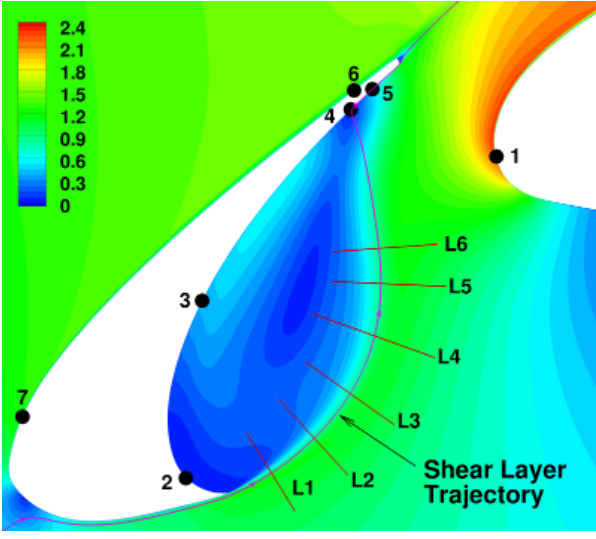

(a) $\operatorname{Span}=2: 9 \mathrm{c}$

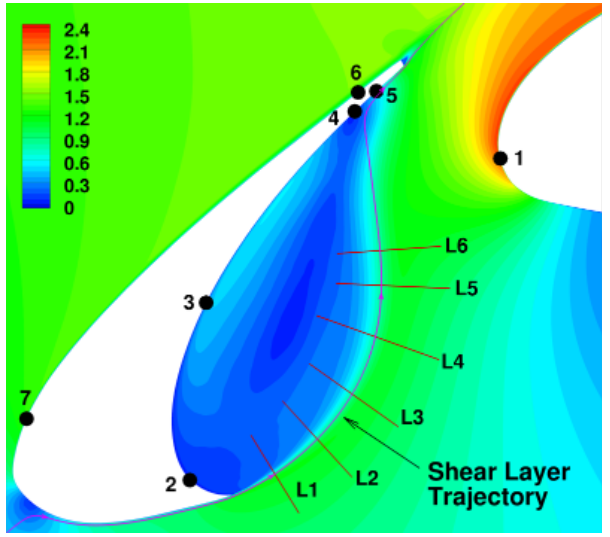

(b) $\mathrm{Span}=\mathrm{c}=9$

Figure 6. Magnitude of the planar velocity, $\left|\mathrm{V} * \mathrm{~V}_{0}^{*}\right|=\mathrm{U}_{0}$, around the slat averaged both temporally and in the spanwise direction. The lines L1-L6 define normal cuts through the shear layer, and the pressure spectra is examined at the surface points 1-6.

A comparison of the flow on the lines shown in Fig. 6 provide a more quantitative comparison of the mean state. The values of the time-averaged spanwise vorticity (normalized by $\left.c / U_{o}\right)$ and streamwise velocity $\left(<u^{*}>/ U_{o}\right)$ on the five line cuts L1, L2, L3, L4 and L6 shown in Fig. 6 are plotted in Fig. 7. The abscissa is the streamwise coordinate $x$, so the starting point for each line cut is slightly different. Here, the short span result exhibits slightly higher vorticity levels close to the slat cusp, but much less discrepancy is observed in the $U$ velocity. However, the slopes of the $U$ velocity (and $V$ velocity which are not shown) across the shear layer are slightly higher for the shorter span simulation, which gives rise to the higher vorticity levels. The vorticity tends to be a sensitive parameter because it involves spatial derivatives of the velocities.

The contours of the 3-D turbulence kinetic energy (TKE), $\frac{1}{2}\left(<u^{\prime} u^{\prime}\right\rangle+\left\langle v^{\prime} v^{\prime}\right\rangle+\left\langle w^{\prime} w^{\prime}\right\rangle$ ), normalized by the square of the streamwise velocity, $U_{o}^{2}$, also reveal some differences as seen in Fig. 8 . Note that these contours represent the resolved portion of the turbulent fluctuations as they do not include any contributions from the portion calculated by the turbulence model. The thin region of high 3-D TKE near the reattachment point and extending to the 
trailing edge is larger in the short span calculation with periodic boundary conditions. The elevated 3-D TKE levels in this region are caused by instabilities in the cove shear layer being rapidly distorted as they approach the underside of the slat. ${ }^{11}$ However, the TKE levels in the section of the shear layer near the cusp are higher for the span $=2.9 \mathrm{c}$ case. In this region, the shear layer is relatively thin, and the most unstable modes are at high frequency. The smaller time step in the new calculation may be better resolving these high frequencies, or it could be something about the mean flow that is promoting a faster initial growth of the instabilities. Unsteady flow features that recirculate in the cove seem to be less likely to approach the slat in the longer span calculation as the dark blue region on the underside of the slat (between points 2 and 3 in Fig. 6) indicates.

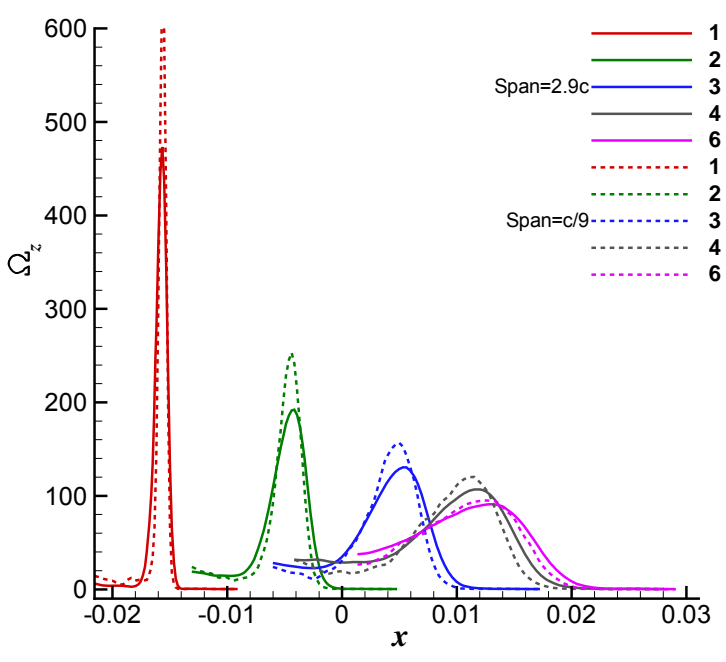

(a) Z Vorticity

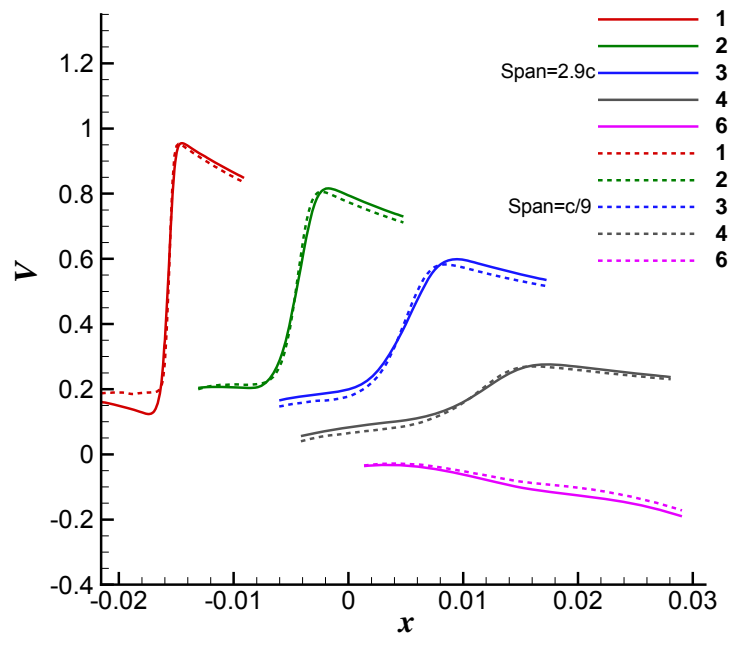

(b) U Velocity

Figure 7. Mean values on line cuts L1-L6 shown in Fig. 6.

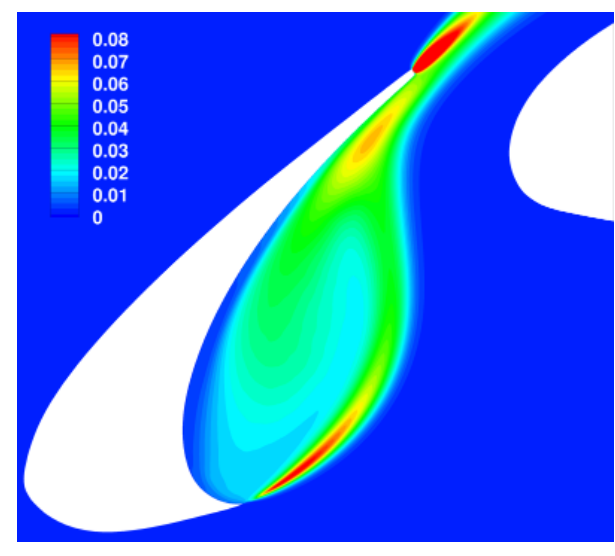

(a) $\mathrm{Span}=2: 9 \mathrm{c}$

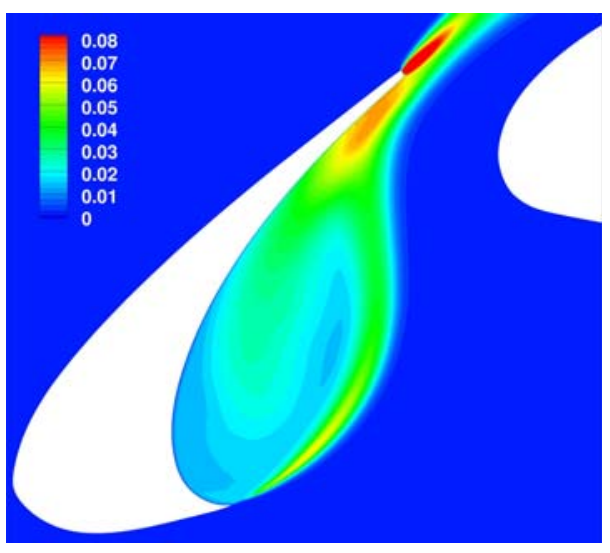

(b) Span $=c=9$

Figure 8. 3-D Turbulence kinetic energy normalized by $U_{0}^{2}$ around the slat averaged both temporally and in the spanwise direction.

So far, only time-averaged quantities have been presented, but the instantaneous view of isosurfaces of the streamwise vorticity $\Omega_{x}$ in Fig. 9 gives an indication of the scales being resolved by the simulations. The view is from below the high-lift system looking at the slat, and the spanwise extent of the shaded high-lift system is $c / 9$ in both figures. Both simulations exhibit spanwise structures that develop in the shear layer near the cusp but gradually break up and then are rapidly distorted near the reattachment point. The red observed upstream of the trailing edge is indicative of the compression occurring in this region. Streamwise structures dominate the flow past the reattachment point and past the trailing edge. In the simulation with a span of $2.9 c$, the structures outside of the center region are sparse because the spanwise spacing is too large to support the fine spanwise scales seen in the central region. Far outside of the core, the flow becomes very two-dimensional in nature. Although not readily apparent in the figure, the flow is aperiodic across 
the span in Fig. 9(a), whereas it is periodic in Fig. 9(b). The spanwise vorticity is compared in Fig. 10 where, outside of the core region in the longer span calculation, the spanwise rollers are seen to persist relatively intact as they move downstream instead of being broken up by smaller 3-D scales. Inside of the central region, the features are again seen to be quite similar between the two simulations.

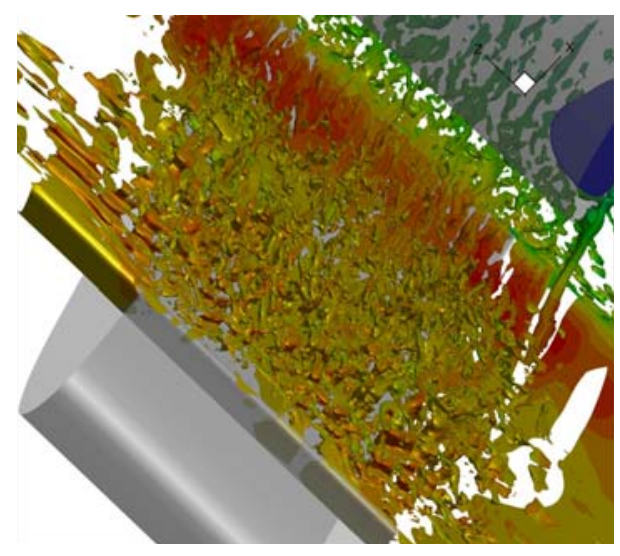

(a) $\mathrm{Span}=2: 9 \mathrm{c}$

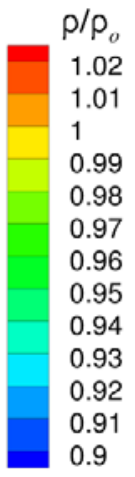

0.9

Figure 9. Isocontours of the instantaneous streamwise vorticity $(x=40)$ colored by density. View is from underneath the high-lift system, looking at the slat.

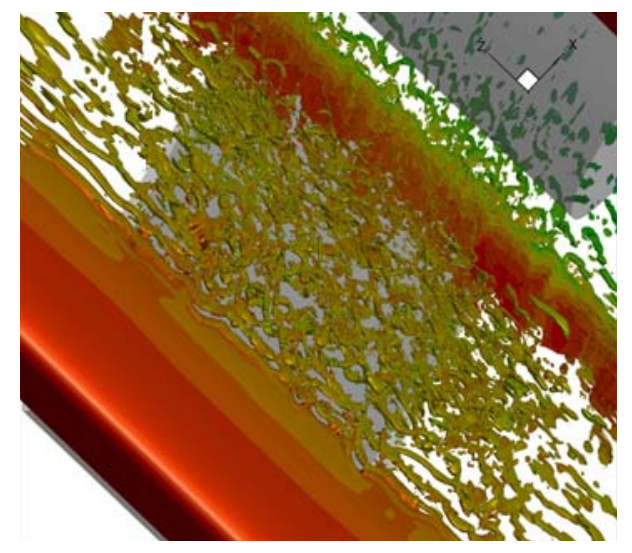

(a) $\operatorname{Span}=2: 9 \mathrm{c}$

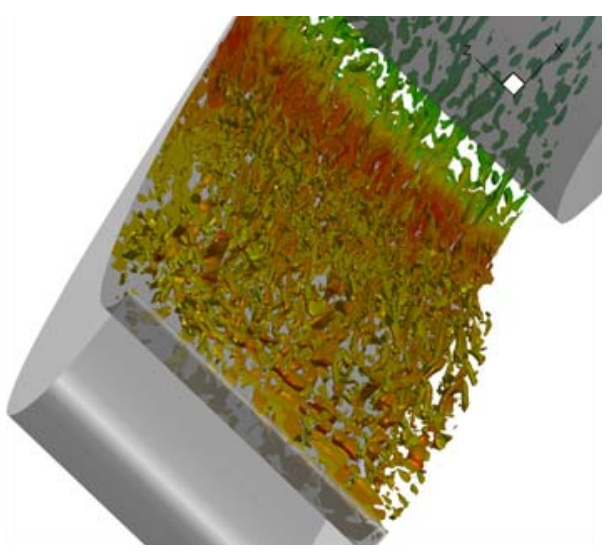

(b) $\mathrm{Span}=\mathrm{C}=9$

Figure 10. Isocontours of the instantaneous spanwise vorticity $(z=75)$ colored by density. View is from underneath the high-lift system, looking at the slat.

The power spectral density (PSD) and spanwise correlation $\left(R_{p p}\right)$ of the surface pressure at the seven points identified by black circles in Fig. 6 are presented in Fig. 11. The power spectral density is plotted against the Strouhal number, $S t=f^{*} c_{s} / U_{o}$, based on the streamwise velocity $U_{o}$, and the slat chord, $c_{s}$. The time histories at the points from the simulations were segmented into seven blocks, Hanning windowed, and then run through an FFT. These seven autospectral results were then averaged, as well as an average over $c / 9$ of the span. The spectra are similar between the two simulations except for point 3 where they diverge at higher frequencies. This point is in the recirculation zones where differences were observed in the mean velocities and TKE. The levels at this point are very low compared with the region near the reattachment (points 4 and 5), so it is not likely to have a big impact on the radiated noise. The large spectral hump between $S t=15-25$ is caused by shedding from the slat trailing edge. The tone near $S t=7.4$ in the $c / 9$ span results at points 1,2,6 and 7 corresponds to an acoustic wave whose wavelength equals the spanwise width $c / 9$ of the periodic domain. In the simulation, an instability developed at this frequency that eventually overwhelmed the entire solution. Other than the appearance of the peak at this frequency, the sampled data from the simulation appears to be free of other artifacts of the instability. One of the reasons for using the longer span was to alleviate this instability, and it appears to have successfully suppressed any extraneous tones.

Points 6 and 7 on the upper surface of the slat (as shown in Fig. 6) were chosen in an attempt to isolate the propagating 
acoustic waves. Because the simulations employ RANS in the near-wall region, they do not resolve any boundary layer instabilities or turbulence there. The absence of any separation or shear layers on the suction side of the slat leaves the region devoid of hydrodynamic type features, making the acoustic signals that are typically buried by the signatures of other flow features relatively easy to see. The spectra at these points has a broadband component between $S t=1-3$ that is generally associated with slat noise, but also a series of narrowband peaks at comparable Strouhal numbers. The source of the peaks is still under investigation; but they are fairly robust, appearing in simulations at different Reynolds numbers and with a spanwise flow. They have also been observed in experiments ${ }^{7}$ conducted at Reynolds numbers similar to the 1.7 million used in these simulations. However, the simulations have been run in a fully turbulent manner, which may not be entirely equivalent to the experiments where most of flow around the slat is laminar. Several theories exist as to the source of these tones, but most postulate some sort of resonance similar to Rossiter modes in shallow cavities. $^{7,24}$

An overall indication of the $3 \mathrm{D}$ character of the flowfield can be ascertained from the spanwise correlation of the surface pressure fluctuations,

$$
R_{p p}(\Delta z)=\lim _{T \rightarrow \infty}{ }_{0}^{\mathrm{Z}_{T}} p(z, t) p(z+\Delta z, t) d t / p_{r m s}^{\prime 2}
$$

as a function of the spanwise separation distance, $\Delta z$. For the simulation with a span of $c / 9$, only the correlation for separation distances up to $c / 18=0.0555$ is shown as the periodicity in the spanwise direction causes the correlation from 0.0555 to 0.1111 to be the mirror image of that from 0 to 0.0555 . In the longer span calculation, the correlation is meaningful across the entire domain, but only the correlation from the center $c / 9$, where the spanwise grid spacing is uniform, is shown. The drop in the correlation with spanwise separation at points 1-5 is similar between the two simulations. However, the correlation at the two points on the upper surface of the slat behave differently. At point 6 , $R_{p p}$ in the longer span calculation drops to near zero, but it plateaus near 0.4 in the periodic case. Although the spectrum at this point is relatively similar in shape to that at point 7, the difference in correlation coefficient is too large to be solely caused by the propagation of an acoustic wave between the two points. Therefore, some evanescent waves are likely to be present in the signal at point 6 , which is not too surprising given its proximity to the trailing edge of the slat. The correlation at point 7 is much higher for both simulations and very nearly unity for all separation distances in the periodic case. This indicates that for most of the energy at this point, the fluctuations are nearly uniform in the spanwise direction.

Another way to look at the spanwise decorrelation is through the coherence,

$$
\gamma^{2}(f)=\frac{\left|G_{p_{1} p_{2}}(f)\right|^{2}}{G_{p_{1} p_{1}}(f) G_{p_{2} p_{2}}(f)}
$$

where $G_{p_{1} p_{2}}(f)$ is the cross-spectral density function between the pressure at two spanwise locations indicated by 1 and 2. The coherence allows one to examine individual frequencies and ascertain the behavior of different phenomena such as the low-frequency, broadband component of slat noise and the high-frequency shedding from the slat trailing edge. The coherence for all frequencies and separation distances is presented in the form of contour maps in Figs. 12-14. The Strouhal number is the abscissa, and the separation distance is the ordinate. The coherence is given by the color which goes from white (no coherence) to blue (fully coherent). Again, for the periodic case, the coherence is only plotted for separation distances up to half the span $(c / 18=0.0555)$. At point 1 in Figs. 12(a) and (b), coherence levels of up to 0.5 are seen for separation distances up to 0.04 . This point is on the nose of the main element and the influence from the hydrodynamic features in the cove is relatively weak. There are slight discontinuities in the coherence for the periodic case at $S t=7.4$ and $S t=14.8$, which correspond to acoustic waves with one and two wavelengths across the span, respectively. Although the longer span calculation does not exhibit this behavior, it also has coherence levels at low frequency that are relatively high compared to those seen at probe locations within the cove (Figs. 12(c)-(f) and Figs. 13(a)-(d)) where the coherence drops off rapidly with spanwise distance except for frequencies corresponding to the trailing edge shedding $(S t=17-25)$, which is not spatially well-resolved in the present simulations. Enhanced coherence at $S t=7.4$ is observed in both simulations at Point 2 (Figs. 12(c) and 12(d)) indicating that there is some phenomena occurring at that frequency that is potentially unrelated to the span in the simulation. In the periodic case, it gets amplified and eventually overwhelms the solution, but even in the longer span calculation, there is some enhanced noise at this frequency as will be seen when the radiated noise is presented.

The coherence at point 6 (Figs. 13(e) and 13(f)) is similar to that at point 1, but now some higher coherence is observed at the frequencies corresponding to the low-frequency cavity tones $(S t=1.6-6)$. However, at point 7 (Fig. 14) the true nature of the radiating acoustics is revealed. In the periodic case, the coherence is unity for $S t<7.4$, indicating that the acoustic waves are completely two-dimensional. The coherence has additional jumps at $S t=14.8$ and 22.2 , but 


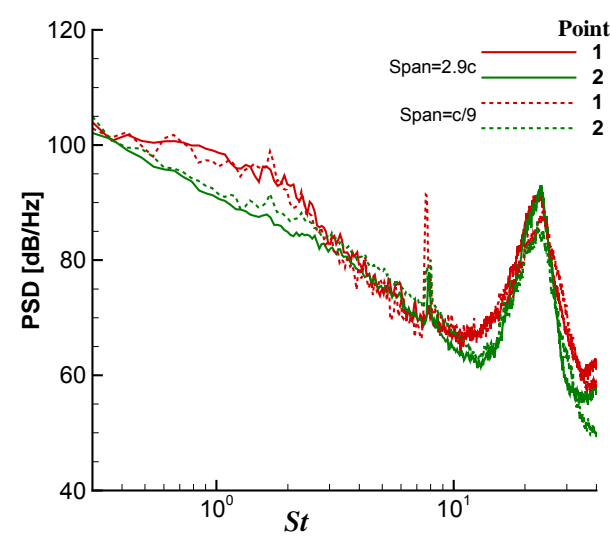

(a) Spectra

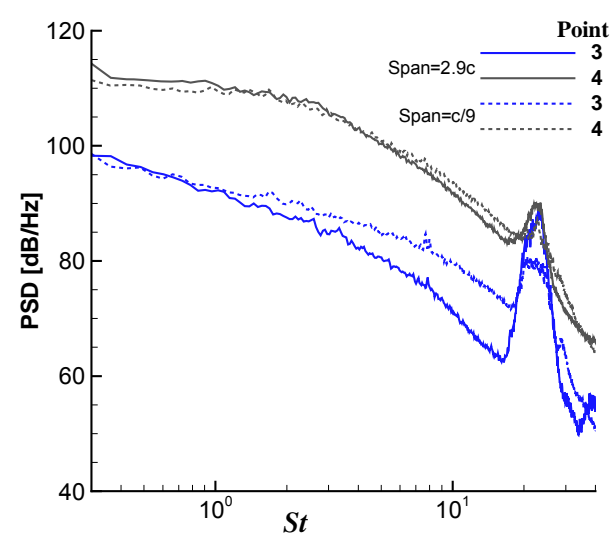

(c) Spectra

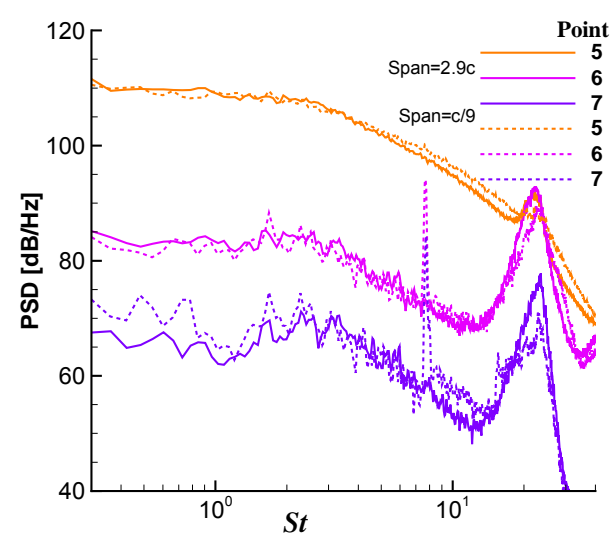

(e) Spectra

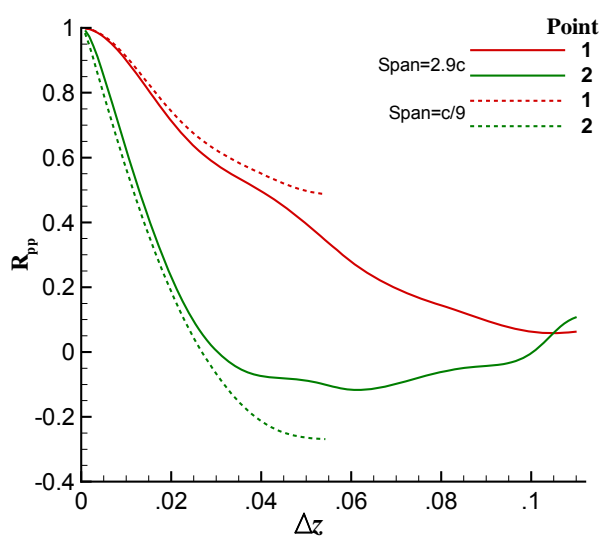

(b) Correlation

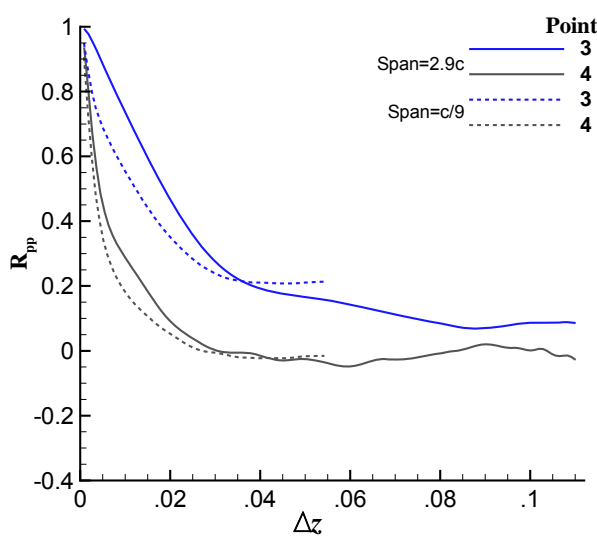

(d) Correlation

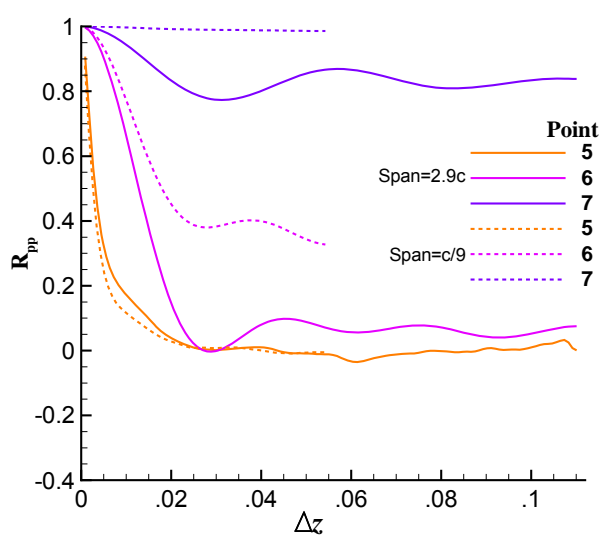

(f) Correlation

Figure 11. Pressure spectra and correlation at the seven surface points in Fig. 6.

they are not as significant as the first one. With the longer span, the coherence is relatively high at low-frequencies, but there is some spanwise variation at all frequencies. Clearly, the spanwise extent and the boundary conditions employed in the simulations have a strong impact on the acoustics that is not readily apparent from the behavior in the cove. The spanwise coherence of the pressure at points off the body behave similarly to that at point 7 . 


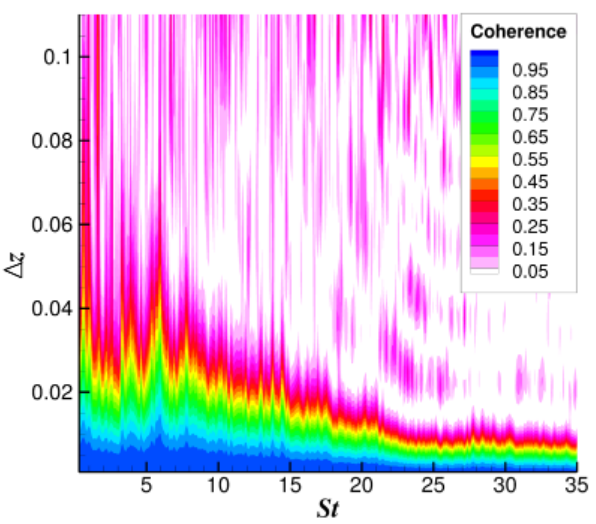

(a) Span=2:9c, Point 1

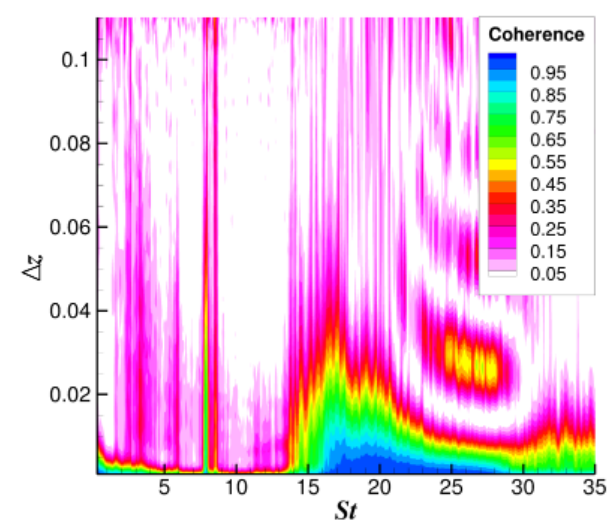

(c) Span=2:9c, Point 2

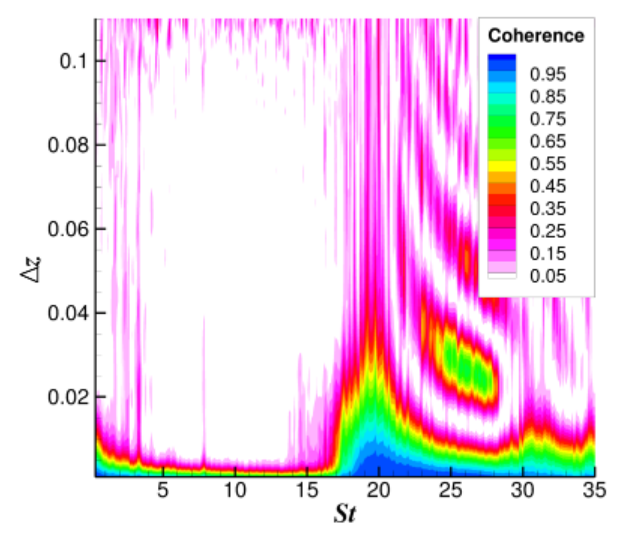

(e) Span=2:9c, Point 3

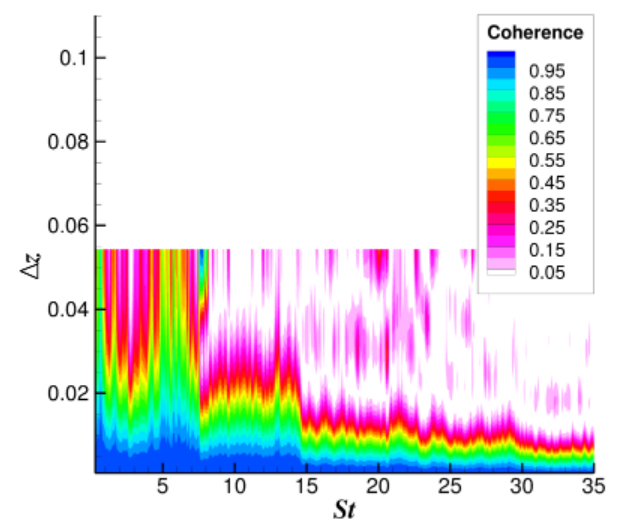

(b) Span=c=9, Point 1

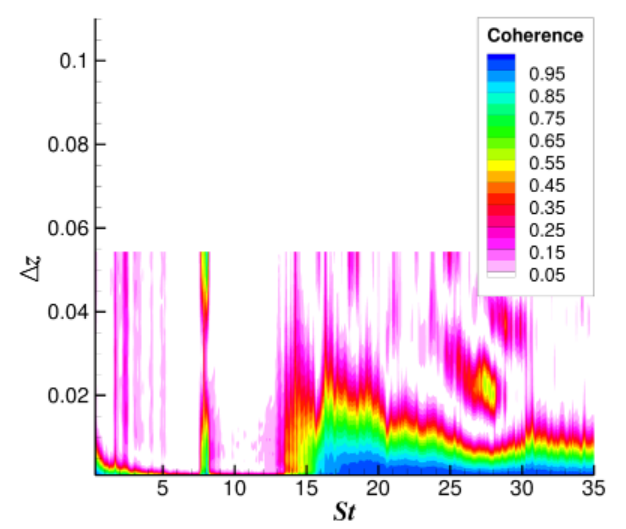

(d) Span=c=9, Point 2

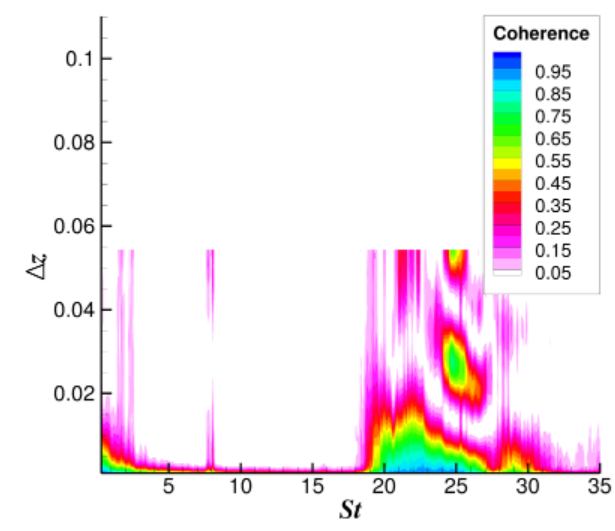

(f) Span=c=9, Point 3

Figure 12. Coherence at points 1-3 in Fig. 6.

\section{Far-Field Acoustics}

The far-field noise was calculated using the Ffowcs Williams-Hawkings (FW-H) equation ${ }^{25}$ frequency-domain solver described by Lockard. ${ }^{26}$ Calculations were also performed with a time-domain solver, ${ }^{26}$ and the directivity and spectral results were in agreement with those shown here from the frequency-domain solver. A grid and time step study was performed with the frequency-domain code, and using every other spatial and temporal point did not change the results. 


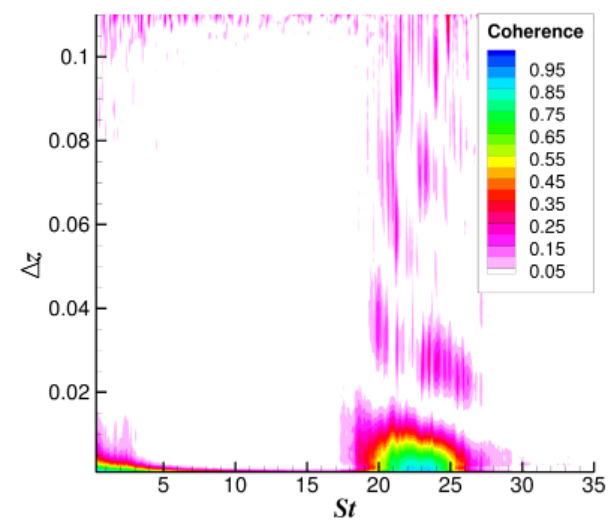

(a) Span=2:9c, Point 4

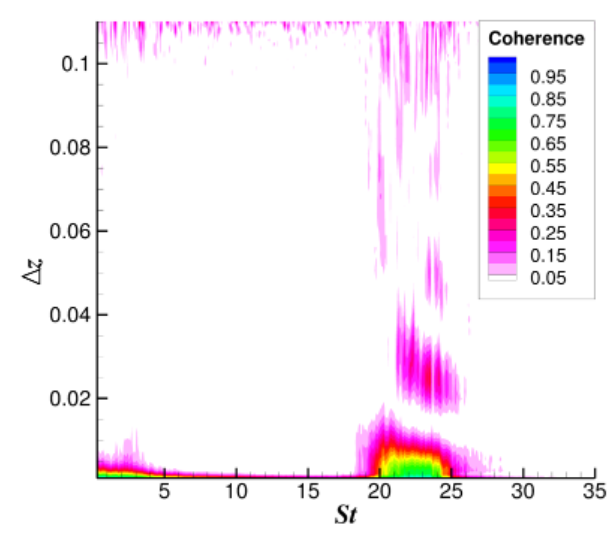

(c) Span=2:9c, Point 5

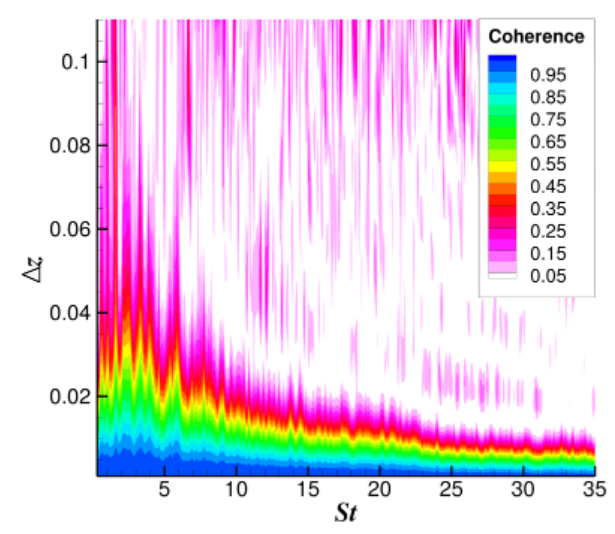

(e) Span=2:9c, Point 6

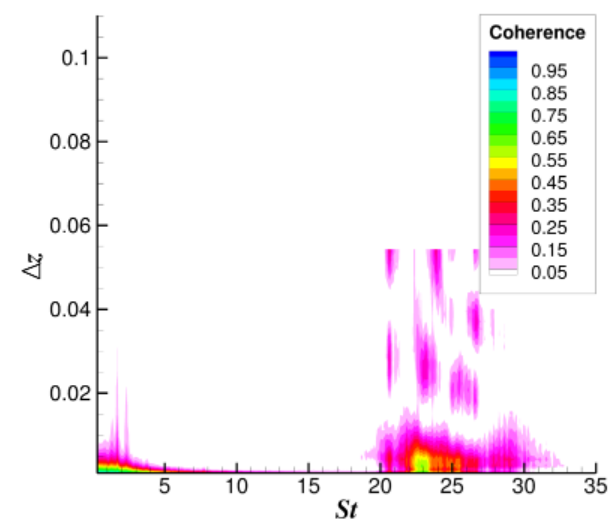

(b) Span=c=9, Point 4

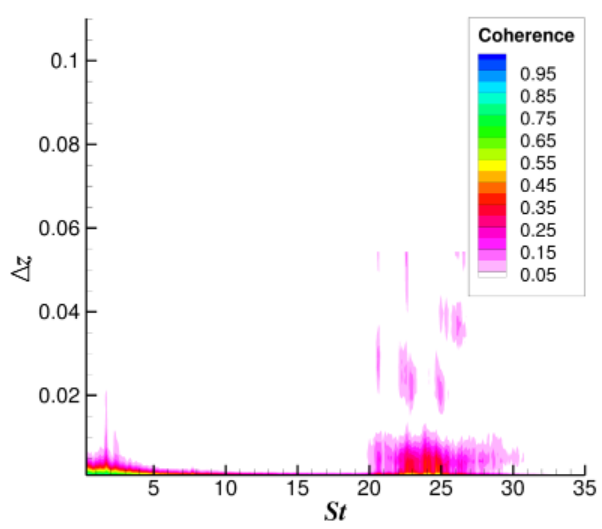

(d) Span=c=9, Point 5

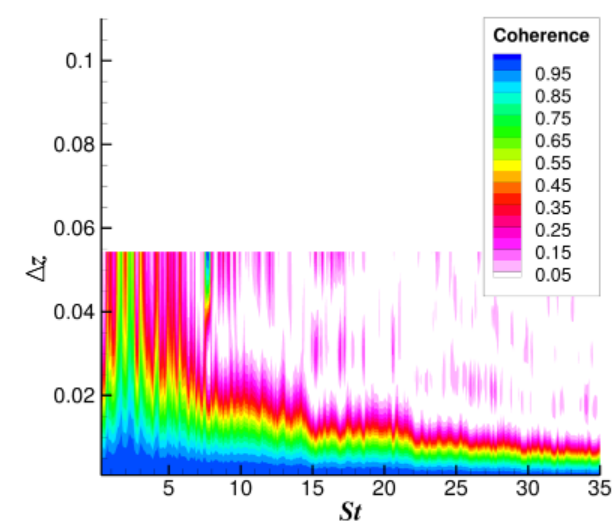

(f) $S p a n=c=9$, Point 6

Figure 13. Coherence at points 4-6 in Fig. 6.

Unsteady flow data from the CFD calculations was recorded on the solid surfaces, with the data extracted over the full span used in the CFD calculation. However, various subsets of the spanwise data were used in the FW-H calculations. The noise predictions are for spanwise segments $L_{s}$ of $18.5 \%, 37 \%, 74 \%$, and $148 \%$ of the slat chord, $c_{s}$ (or $c / 36, c / 18$, $c / 9,2 c / 9$, respectively). In all cases, the observers were located at the spanwise middle of the section used in the noise calculation. The $37 \%$ segment ( $c / 18$, approximately 1 in) corresponds with what has been examined previously ${ }^{15}$ for the 


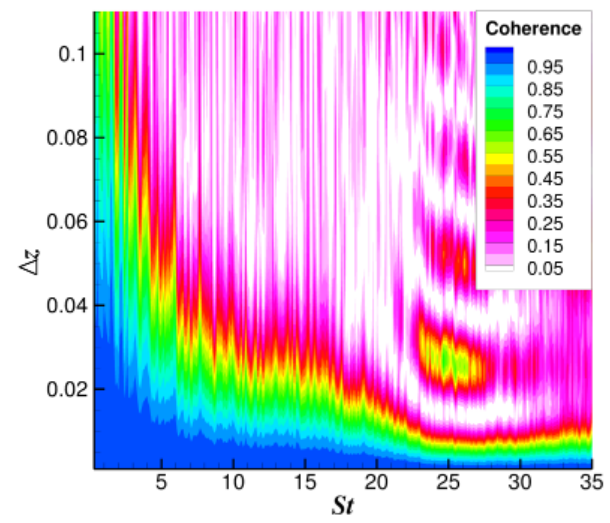

(a) Span=2:9c, Point 7

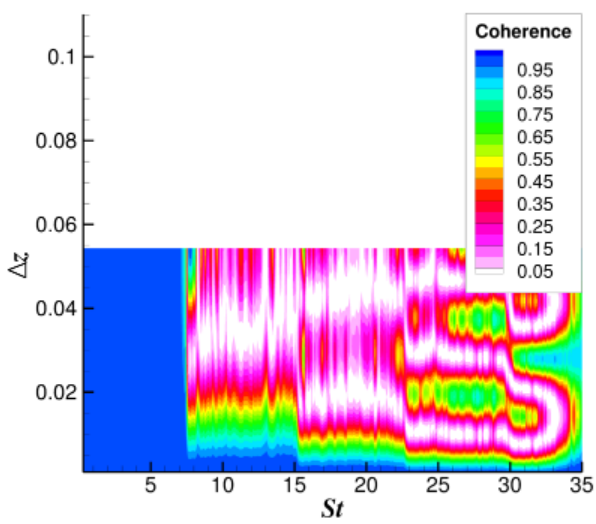

(b) Span=c=9, Point 7

Figure 14. Coherence at point 7 in Fig. 6.

periodic problem as that only uses half of the spanwise domain in the FW-H calculations, which should avoid artificial interference effects caused by the periodic nature of the simulations. The $c / 9$ segment is the full extent of the periodic simulation, so $L_{s}^{*}=2 c / 9$ can only be calculated for the case with a span of $2.9 c$. The temporal record of 39,000 time steps was divided into seven separate segments, where successive segments were overlapped by $50 \%$ and windowed with a Hanning filter. Each temporal segment was run through the FW-H solver independently, and the seven individual results were then averaged.

The directivity for Strouhal numbers between $S t=0.3-7.3$ is shown in Fig. 15. The upper frequency is just below $S t=7.4$ that corresponds to the tone that overwhelmed the periodic calculation, and the lower frequency of 0.3 excludes some of the oscillations that are not adequately represented in the short time records available from the simulations. The temporal length is sufficient to resolve $S t=0.3$ with approximately six periods. The distance from the slat trailing edge to the observers is 10 chords. As is evident in Fig. 15(a), the shape of the directivity is relatively insensitive to the spanwise extent used in the FW-H, but the amplitude is increasing monotonically as $L_{s}^{*}$ is increased. The data for various $L_{s}^{*}$ has been collapsed in Fig. 15(a) by scaling $\left|p^{\prime}\right|$ by 1.8 for each doubling of $L_{s}^{*}$ except between $c / 9$ and $2 c / 9$ where 1.3 is used. The case with $L_{s}^{*}=c / 9$ is used as the reference. The collapse is quite good, with only modest discrepancies at certain angles. Interestingly, using the full extent of the periodic domain produced results that scale perfectly with those of the two shorter domains. If the spanwise correlation of the hydrodynamics had any influence on the noise, then the perfect correlation between the two ends of the full span should have had some influence on the results. As was observed through the coherence analysis in Fig. 14(b), acoustic waves are fully correlated across the entire span for this frequency range, so there is no variation in spanwise coherence to have an effect on the FW-H calculation. The implication is that the FW-H is not determining the acoustic source based on the total fluctuations, but is instead filtering out the non-radiating portion of the signal and extending the underlying acoustic signal to the far field.

According to the theory of Kato et al. ${ }^{27}$ and Seo et al., ${ }^{28}$ which provides a formula for adjusting the levels obtained from a short span to account for a longer span, the scaling factor for doubling the span asymptotes to 2.0 when the coherence length is greater than the length of the segment being extended. The discrepancy between the optimal scaling of 1.8 for the simulations and the 2.0 predicted by the formula is still under investigation but may be related to some of the assumptions used to derive the relation. The coherence length, $L_{c}$, is defined in terms of an exponential representation of the coherence as $\gamma^{2}(f)=\exp \left(-2 z^{2} / L_{c}^{2}\right)$. For the periodic case, the spanwise coherence of the acoustic waves with $S t<7.4$ is definitely longer than $L_{s}$. Even though the spanwise coherence length of the acoustics is smaller in the aperiodic case, the coherence for $S t<7.4$ is still quite high and $\gamma^{2}(f)$ does not drop below 0.5 until the $\Delta z$ between the points exceeds $c / 18$. Hence, the directivity is scaling similarly to the periodic case until $L_{s}^{*}$ exceeds $c / 9$. For the scaling between $L_{s}=c / 9$ and $2 c / 9$, the coarseness of the grid outside of the core $c / 9$ region may be playing some role in the scaling, but the grid is still quite fine for the wavelengths of the acoustic waves with $S t<7.4$. The $L_{s}^{*}=2 c / 9$ case is including enough span in the FW-H calculation so that the frequencies corresponding to the peak in spectrum are undergoing enough decorrelation to influence the far-field noise calculation; however, the noise generation that should be occurring outside of the core region is probably being underpredicted. The minimum scaling factor predicted by the theory is $\sqrt{2}$ and occurs when $L_{c} / L_{s}<0.56$. The optimal scaling factor of 1.3 found in the simulation is slightly less than the theoretical minimum, which may indicate that the grid is too coarse or be related to the assumptions in the theory. 


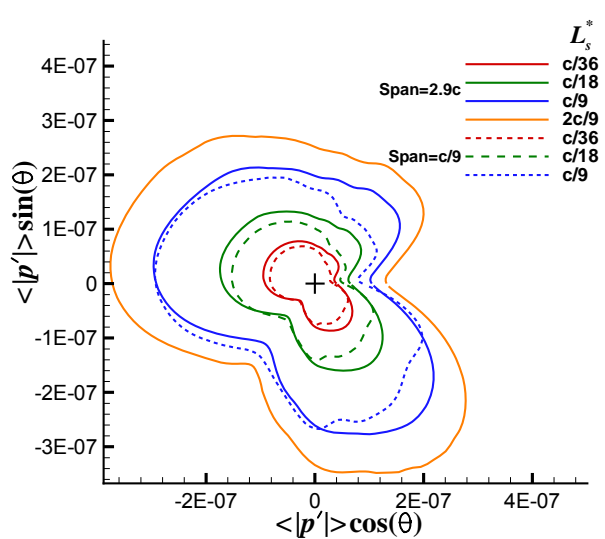

(a) Unscaled

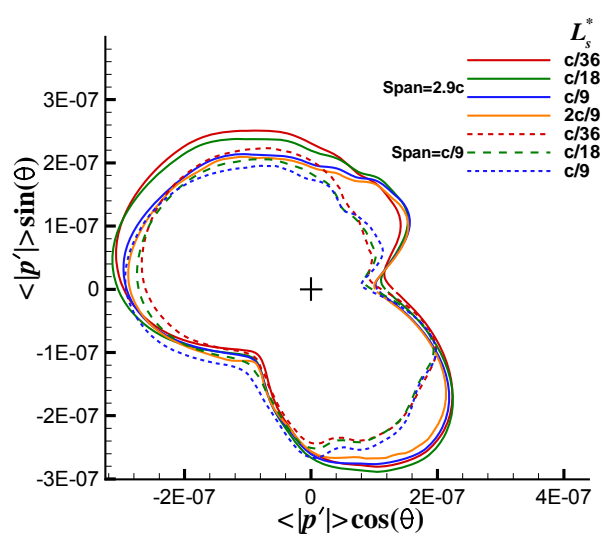

(b) Scaled

Figure 15. Directivity for $S t=0.3-7.3$ at a radius of $10 \mathrm{c}$. The scaled results employ a scaling factor for $\left|p^{\prime}\right|$ of 1.8 for each doubling of $L_{S}^{*}$ except between $2 c=9$ and $c=9$ where 1.3 is used.

The power spectral density of the pressure at $290^{\circ}$ counterclockwise from the positive $x$ axis is presented in Fig. 16. The $x$-axis is aligned with the chord of the airfoil, not the freestream direction. The current data represent the average of seven realizations with a bin width of $\Delta S t=0.074$ at $U_{\infty}=U_{o}=0.17$, corresponding to $48 \mathrm{~Hz}$. For both calculations, the PSD at lower frequencies increases nearly uniformly with $L_{s}^{*}$, but there is very little effect between $S t=15-25$ where the broad hump associated with the trailing-edge shedding resides. The frequency dependence of the levels on both $L_{s}$ and $L_{c}$ is apparent in these results. One may notice that the tone at $S t=7.5$ disappears for $L_{s}^{*}=c / 9$ in Fig. 16(b). This is because the entire spanwise domain was used in the FW-H calculation, and this tone is essentially a standing mode with a node at the center of the domain where the observers are located.

The spectra for $L_{s}^{*}=c / 18$ from the two simulations are directly compared in Fig.16(c). The periodic case with a span of $c / 9$ has more well defined peaks whereas the spectrum from the aperiodic case is a little more filled in with slightly higher levels between $S t=2-6$. However, the peak at $S t=7.4$ is much weaker, and the tone at $S t=0.75$ is completely gone. This peak was associated with the cove in the main element where the flap nests, and the reason for the dependence on the span is unclear. However, apart from the above mentioned differences, the general broadband character of the spectra is similar between the two calculations.

The spectra for the longer span case are scaled similarly to the directivity scaling in Fig. 15 and plotted as Fig.17(a). Once again, $\left|p^{\prime}\right|$ is scaled by 1.8 for each doubling of $L_{s}^{*}$ except between $c / 9$ and $2 c / 9$ where 1.3 is used. The collapse is nearly perfect up to $S t=4$, after which the curves diverge. Because the majority of the energy resides in frequencies below $S t=4$, the imperfect scaling at higher frequencies does not affect the directivity, which is why the good collapse was observed in Fig. 15(b). The spectral collapse for the periodic case (Fig. 17(b)) is very good up to $S t=7.4$, as would be expected based on the perfect spanwise coherence up to that frequency.

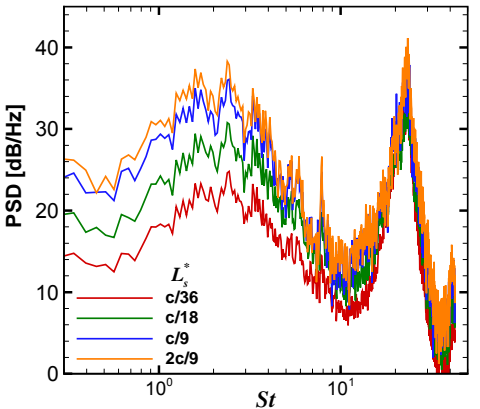

(a) $\mathrm{Span}=2: 9 \mathrm{c}$

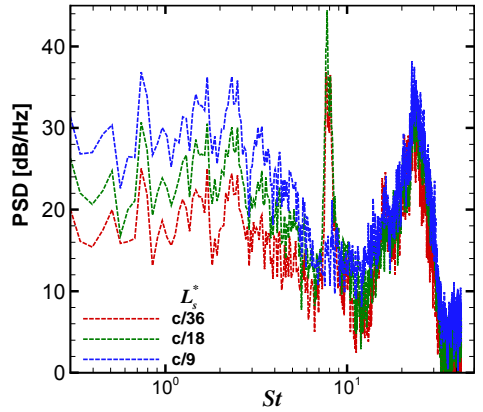

(b) Span $=c=9$

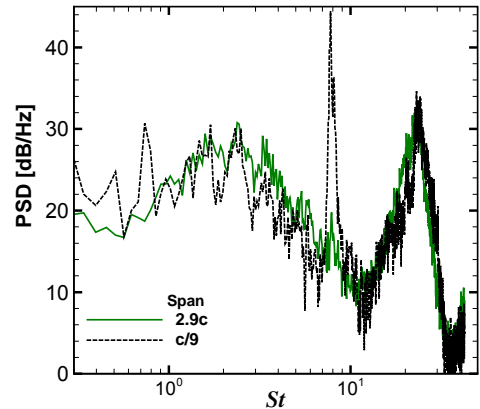

(c) $L_{\mathrm{s}}^{*}=\mathrm{C}=18$

Figure 16. Spectra at a radius of $10 \mathrm{c}$ and $290^{\circ}$ from the positive $x$ axis. 


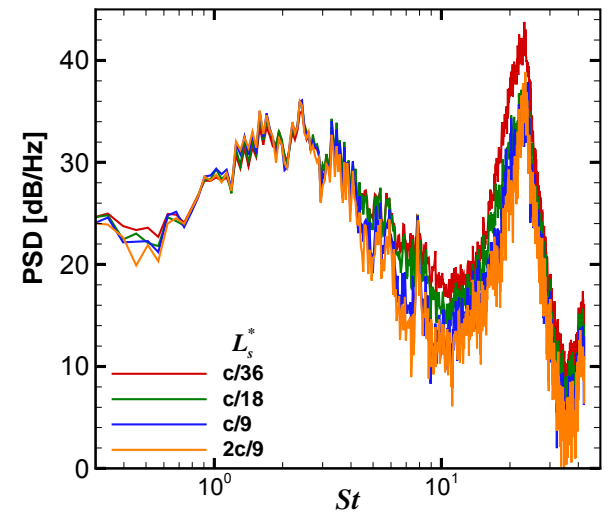

(a) Scaled, Span $=2: 9 \mathrm{c}$

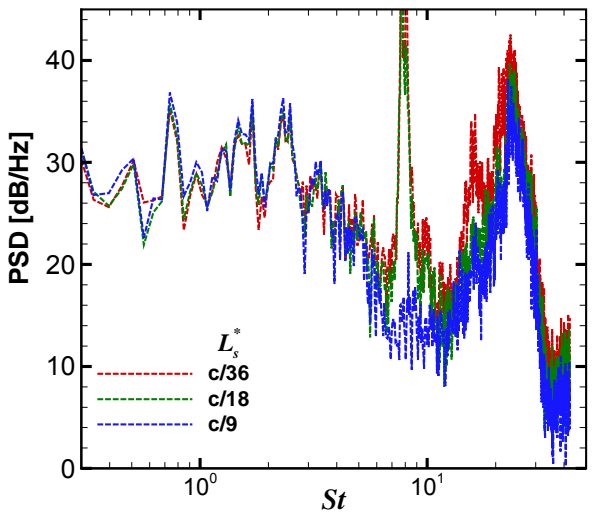

(b) Scaled, Span $=c=9$

Figure 17. Spectra at a radius of $10 \mathrm{c}$ and $290^{\circ}$ from the positive $x$ axis. The scaled results employ a scaling factor for $\left|p^{\prime}\right|$ of 1.8 for each doubling of $L_{s}^{*}$ except between $2 c=9$ and $c=9$ where 1.3 is used.

\section{Conclusions}

This paper has investigated the influence of the spanwise domain extent and periodicity on unsteady simulations of the flow around a slat. A new calculation with a total span of $2.9 c$ was conducted by adding a stretched mesh outside of the core $c / 9$ previously used with periodic boundary conditions. Slip-wall conditions were used at the ends of the $2.9 \mathrm{c}$ domain. The first and second moment statistics between the simulations are similar, with only slight variations in the mean flow. The spectra at various points around the slat are also similar between the two simulations, and even the spanwise correlation is similar, except for points on the upper surface of the airfoil that are more influenced by acoustic waves than hydrodynamic flow features. An examination of the coherence demonstrated that the spanwise coherence drops rapidly for the hydrodynamic features, but acoustic waves in the periodic case are perfectly coherent across the entire span up to a frequency of $S t=7.4$. The periodicity introduces duct-like behavior, and only two-dimensional waves can propagate up to that frequency. Eventually, the periodic simulation blew up when an instability at that frequency grew without bound. This instability was suppressed in the simulation with a span of $2.9 c$, and the spanwise coherence varied with the spanwise separation distance for all frequencies. However, the low frequencies dominating the broadband component of slat noise were still found to be very coherent. The far-field noise was calculated using a frequency-domain solution of the Ffowcs Williams-Hawkings (FW-H) equation, ${ }^{25}$ and calculations were performed using several spanwise segments, all of which were long compared to a hydrodynamic length scale but short compared to the coherence length of the dominant acoustic frequencies. In the periodic case, the noise scaled directly with the length of the span, which means that the spanwise coherence did not influence the result. This implies that it is the coherence of the underlying acoustic waves and not the coherence of the hydrodynamics that is important. In the long span calculation, the influence of the spanwise coherence was observed when using a spanwise segment larger than the entire $c / 9$ domain of the periodic case. Unfortunately, the peak in the broadband component of slat noise is between $S t=1-3$, and the short span calculation with periodic boundary conditions cannot give us the coherence information that is needed to attempt to scale the results up to a realistic span length. Indeed, the span would need to be increased by at least a factor of eight to get meaningful results for the important frequency range. Even though the short-span simulation with periodic boundary conditions cannot provide the information needed to estimate the amplitudes for a realistic wing, the directivity and spectral shape information should be representative. Furthermore, changes in noise caused by configuration changes are likely to be meaningful. Although the longer span calculation appears to have more faithfully reproduced the physics, the effect of the stretched mesh outside of the core region needs more thorough investigation. Experimental data for the acoustics from the $30 \mathrm{P} / 30 \mathrm{~N}$ should be available soon, which will provide the opportunity to test whether formulas for extending the noise from short spans to longer ones can be employed successfully with the available computational data. Such comparisons may be simpler at higher angles of attack that weaken the very coherent, narrowband peaks that are observed in the same low-frequency range as the broadband component of slat noise.

\section{Acknowledgments}

This work was supported by the NASA Advanced Air Transport Technology (AATT) Project. The authors gratefully acknowledge the assistance of Mr. M. R. Wiese from Vigyan, Inc., who generated the baseline computational grids used during the present study. 


\section{References}

${ }^{1}$ Hayes, J. A., Horne, W. C., Soderman, P. T., and Bent, P. H., "Airframe Noise Characteristics of a 4.7\% Scale DC-10 Model," AIAA Paper 1997-1594, 1997.

${ }^{2}$ Storms, B. L., Ross, J. C., Horne, W. C., Hayes, J. A., Dougherty, R. P., Underbrink, J. R., Scharpf, D. F., and Moriarty, P. J., "An Aeroacoustic Study of an Unswept Wing with a Three-Dimensional High Lift System," NASA TM 112222, February 1998.

${ }^{3}$ Dobrzynski, W., Nagakura, K., Gehlhar, B., and Buschbaum, A., "Airframe Noise Studies on Wings with Deployed High-Lift Devices," AIAA Paper 1998-2337, 1998.

${ }^{4}$ Storms, B. L., Hayes, J. A., Moriarty, P. J., and Ross, J. C., "Aeroacoustic Measurements of Slat Noise on a Three-Dimensional High-Lift System," AIAA Paper 1999-1957, 1999.

${ }^{5}$ Choudhari, M. M., Lockard, D. P., Macaraeg, M. G., Singer, B. A., Streett, C. L., Neubert, G. R., Stoker, R. W., Underbrink, J. R., Berkman, M. E., Khorrami, M. R., and Sadowski, S. S., "Aeroacoustic Experiments in the Langley Low-Turbulence Pressure Tunnel," NASA TM 211432, February 2002.

${ }^{6}$ Mendoza, F. and Brooks, T., "Aeroacoustic Measurements of a Wing/Slat Model," AIAA Paper 2002-2604, 2002.

${ }^{7}$ Terracol, M., Manoha, E., and Lemoin, B., "Noise Sources Generation in a Slat Cove: Hybrid Zonal RANS/LES Simulation and Dedicated Experiment," AIAA Paper 2011-3203, 2011.

${ }^{8}$ Chow, L. C., Mau, K., and Remy, H., "Landing Gears and High Lift Devices Airframe Noise Research," AIAA Paper 2002-2408, 2002.

${ }^{9}$ Jenkins, L. M., Khorrami, M. R., and Choudhari, M. M., "Characterization of Unsteady Flow Structures Near Leading-Edge Slat: Part I. PIV Measurements," AIAA Paper 2004-2801, 2004.

${ }^{10}$ Klausmeyer, S. M. and Lin, J., "Comparative Results from a CFD Challenge Over a 2D Three-Element High-Lift Airfoil," NASA TM 112858, May 1997.

${ }^{11}$ Choudhari, M. M. and Khorrami, M. R., "Effect of Three-Dimensional Shear-Layer Structures on Slat Cove Unsteadiness," AIAA Journal, Vol. 45, No. 9, 2007, pp. 2174-2186.

${ }^{12}$ Pascioni, K., Cattafesta, L. N., and Choudhari, M. M., "An Experimental Investigation of the 30P30N Multi-Element High-Lift Airfoill," AIAA Paper 2014-3062, 2014.

${ }^{13}$ Murayama, M., Nakakita, K., Yamamoto, K., Ura, H., Ito, Y., and Choudhari, M. M., "Experimental Study on Slat Noise from 30P30N Three-Element High-Lift Airfoil at JAXA Hard-Wall Lowspeed Wind Tunnel,” AIAA Paper 2014-2080, 2014.

${ }^{14}$ Lockard, D. P. and Choudhari, M. M., "Noise Radiation from a Leading-Edge Slat," AIAA Paper 2009-3101, 2009.

${ }^{15}$ Lockard, D. P., Choudhari, M. M., and Buning, P. G., "Grid Sensitivity Study for Slat Noise Simulations," AIAA Paper 2014-2627, 2014

${ }^{16}$ Bisek, N. J., "Sidewall Interaction of a Supersonic Flow over a Compression Ramp," AIAA Paper 2015-1976, 2015.

${ }^{17}$ Dawson, D. M. and Lele, S. K., "Large Eddy Simulation of A Three-Dimensional Compression Ramp Shock-Turbulent Boundary Layer Interaction," AIAA Paper 2015-1976, 2015.

${ }^{18}$ Jespersen, D., Pulliam, T., and Buning, P., "Recent enhancements to OVERFLOW," AIAA Paper 1997-0644, 1997.

${ }^{19}$ Thomas, J. L., Krist, S., and Anderson, W., "Navier-Stokes Computations of Vortical Flows Over Low-Aspect-Ratio Wings," AIAA Journal, Vol. 28, No. 2, 1990, pp. 205-212.

${ }^{20}$ Nichols, R., Tramel, R., and Buning, P., "Solver and Turbulence Model Upgrades to OVERFLOW 2 for Unsteady and High-Speed Applications," AIAA Paper 2006-2824, 2006.

${ }^{21}$ Beam, R. and Warming, R., "An Implicit Finite-Difference Algorithm for Hyperbolic Systems in Conservation Law Form," Journal of Computational Physics, Vol. 22, 1976, pp. 87-110.

${ }^{22}$ Shur, M. L., Spalart, P. R., Strelets, M., and Travin, A. K., "A Hybrid RANS-LES Approach with Delayed-DES and Wall-Modelled LES Capabilities," International Journal of Heat and Fluid Flow, Vol. 29, 2008, pp. 1638-1649.

${ }^{23}$ Strelets, M., "Detached Eddy Simulation of Massively Separated Flows," AIAA Paper 2001-0879, 2001.

${ }^{24}$ Deck, S. and Laraufie, R., "Numerical Investigation of the Flow Dynamics Past a Three-element Aerofoil," Journal of Fluid Mechanics, Vol. 732, 2013, pp. 401-444.

${ }^{25}$ Ffowcs Williams, J. E. and Hawkings, D. L., "Sound Generation by Turbulence and Surfaces in Arbitrary Motion," Philosophical Transactions of the Royal Society, Vol. A264, No. 1151, 1969, pp. 321-342.

${ }^{26}$ Lockard, D. P., "A Comparison of Ffowcs Williams-Hawkings Solvers for Airframe Noise Applications," AIAA Paper 2002-2580, 2002.

${ }^{27}$ Kato, C., Iida, A., Takano, Y., Fujita, H., and Ikegawa, M., "Numerical Prediction of Aerodynamic Noise Radiated from Low Mach Number Turbulent Wake," AIAA Paper 1993-0145, 1993.

${ }^{28}$ Seo, J. H., Chang, K. W., and Moon, Y. J., “Aerodynamic Noise Prediction for Long-Span Bodies,” AIAA Paper 2006-2573, 2006. 